\title{
Smith-Purcell radiation emission in aperiodic arrays
}

\author{
J. R. M. Saavedra, ${ }^{1}$ D. Castells-Graells,${ }^{1}$ and F. Javier García de Abajo ${ }^{1,2, \text { * }}$ \\ ${ }^{1}$ ICFO-Institut de Ciencies Fotoniques, \\ The Barcelona Institute of Science and Technology, \\ 08860 Castelldefels (Barcelona), Spain \\ ${ }^{2}$ ICREA-Institució Catalana de Recerca i Estudis Avançats, \\ Passeig Lluís Companys, 23, 08010 Barcelona, Spain
}

(Dated: October 20, 2016)

\begin{abstract}
We study the Smith-Purcell light emission produced by electrons moving parallel to linear aperiodic particle arrays. This constitutes a generalization of this type of phenomenon from periodic to aperiodic structures. Like in the periodic case, the emission is found to exhibit intense features in its angular and frequency distributions, associated with the condition of constructive interference between the contributions arising from different particles in the array. This condition can also be expressed in terms of momentum conservation involving reciprocal wave-vector transfers from the array. We consider two examples of quasiperiodic and hyperuniform aperiodic arrays that allow us to illustrate this idea. Our study opens a new unexplored direction in the interaction of fast electrons with aperiodic arrays characterized by strong features in reciprocal space, which dominate the electron-array interaction.
\end{abstract}

PACS numbers: 41.60.-m,78.60.Hk,42.25.Fx, 


\section{INTRODUCTION}

The interaction of an electron with an inhomogeneous dielectric environment gives rise to transient induced charges and currents that result in the emission of radiation. In particular, the Smith-Purcell (SP) effect ${ }^{1}$ refers to the light emission produced by an electron moving parallel to a periodic grating. As the electron passes close to each successive element along the grating, its evanescent electromagnetic field induces light emission at selected frequencies and directions resulting from a simple condition of constructive interference. This condition is analogous to the Huygens construction, but now also involving the time delay used by the electron to interact with consecutive grating periods. Following the initial demonstration and explanation of the SP effect, 1 subsequent studies have further corroborated the dependence of the emission on electron energy and grating period, ${ }^{2 / 3}$ confirming that it occurs over a

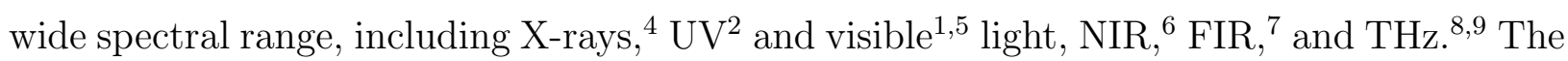
SP effect is the basis of free-electron lasers, 10111 whereby the emission intensity produced a large number of electrons bunched within a small spatial region compared with the emitted light wavelength is proportional to the square of the number of electrons $\frac{812}{14}$. Experimental realizations of the SP effect have focused on periodic structures of different nature, including metallic gratings ${ }^{1115}$, where an interesting interplay takes place between the emitted light and the plasmons supported by the metal surface, as well as dielectric structures $\frac{16}{16}$ and photonic crystals. $17-19$

Strong resonances in reciprocal space lie at the core of SP emission: the emission angles and frequencies are determined by the reciprocal lattice vectors of the periodic lattice with which the electron interacts. One expects that similarly intense emission patterns would result from the interaction with other types of structures, not necessarily periodic, in which strong features are also present in reciprocal space. We explore this extension of the SP effect in this work by first studying the reciprocal-space properties of quasiperiodic ${ }^{20}$ and hyperuniform ${ }^{21}$ one-dimensional arrangements of particles. We find the resulting emission to also display intense features, which we further compare with those of periodic arrays. 


\section{THEORETICAL MODEL}

\section{A. Smith-Purcell emission in linear arrays}

We consider a linear array of $N$ identical particles placed along the $z$ axis at positions $z_{n}$ and an electron moving with constant velocity $v$ parallel to this axis at a distance $b$ from the array, as illustrated by Fig. 1. The electron generates an external evanescent field

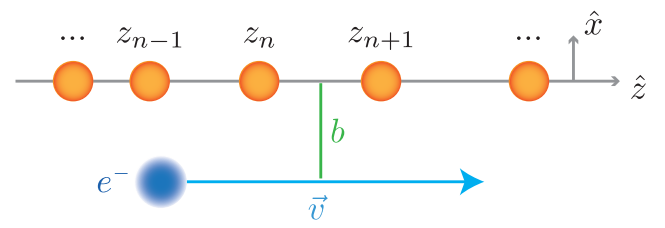

FIG. 1: Schematic representation of the system under consideration. A linear array of spherical particles aligned along the $z$ axis is characterized by the positions of the sphere centers $z_{n}$. An electron moves parallel to the array with constant velocity $v$ at a distance $b$ from the $z$ axis. The array contains $N$ particles with the same diameter $D$.

$\mathbf{E}^{\operatorname{ext}}(z, t)=\int \mathbf{E}^{\operatorname{ext}}(z, \omega) \mathrm{e}^{-\mathrm{i} \omega t} d \omega / 2 \pi$ acting on the particles, whose component of frequency $\omega$ reduces $\operatorname{to}^{22} \mathbf{E}^{\operatorname{ext}}(z, \omega)=\mathrm{e}^{\mathrm{i} \omega z / v} \mathbf{g}(\omega)$, where

$$
\mathbf{g}(\omega)=\frac{2 \mathrm{e} \omega}{v^{2} \gamma}\left[\frac{\mathrm{i}}{\gamma} \mathrm{K}_{0}\left(\frac{\omega b}{v \gamma}\right) \hat{\mathbf{z}}-\mathrm{K}_{1}\left(\frac{\omega b}{v \gamma}\right) \hat{\mathbf{x}}\right]
$$

$\gamma=1 / \sqrt{1-v^{2} / c^{2}}$ is the Lorentz factor, and $\mathrm{K}_{\nu}$ are modified Bessel functions ${ }^{23}$ In what follows, we describe each $\omega$ component independently, as it generates radiation at that frequency when scattered by the array.

For simplicity, we assume spherical particles described through their dipolar polarizability $\alpha(\omega)$, which is related to the electric Mie coefficient $t_{1}^{\mathrm{E}} \operatorname{through}^{22} \alpha(\omega)=3 t_{1}^{\mathrm{E}} / 2 k^{3}$, where $k=\omega / c$ and $k^{\prime}=\sqrt{\epsilon} k$. In particular, we present results for homogeneous spheres of radius $a$ and permittivity $\epsilon$, so that $t_{1}^{\mathrm{E}}=\mathrm{i} /\left(1+\mathrm{i} \Delta_{1}\right)$, where $\Delta_{1}=\left[y_{1}(k a) J\left(k^{\prime} a\right)-\right.$ $\left.\epsilon Y(k a) j_{1}\left(k^{\prime} a\right)\right] /\left[j_{1}(k a) J\left(k^{\prime} a\right)-\epsilon J(k a) j_{1}\left(k^{\prime} a\right)\right], j_{1}$ and $y_{1}$ are spherical Bessel functions, $\stackrel{23}{23}$ $J(\rho)=\rho j_{0}(\rho)-j_{1}(\rho)$, and $Y(\rho)=\rho y_{0}(\rho)-y_{1}(\rho)$. The use of the Mie coefficient instead of an electrostatic expression for $\alpha$ allows us to account for retardation effects in the dipolar response, such as particle resonance redshifts and radiative broadenings. 
The electron field produces a direct induced dipole on each particle $n$ given by $\alpha \mathbf{E}^{\mathrm{ext}}\left(z_{n}, \omega\right)=\alpha \mathrm{e}^{\mathrm{i} \omega z_{n} / v} \mathbf{g}(\omega)$. Interaction among the particles then leads to self-consistent dipoles

$$
\mathbf{p}_{n}=\alpha\left(\mathrm{e}^{\mathrm{i} \omega z_{n} / v} \mathbf{g}+\sum_{n^{\prime} \neq n} \mathcal{G}_{n n^{\prime}} \mathbf{p}_{n^{\prime}}\right),
$$

where the sum yields the electric field produced on particle $n$ by the rest of the particles, mediated by the $3 \times 3$ dipole-dipole interaction matrix

$$
\begin{aligned}
\mathcal{G}_{n n^{\prime}} & =\frac{\mathrm{e}^{\mathrm{i} \theta_{n n^{\prime}}}}{\left|z_{n}-z_{n^{\prime}}\right|^{3}} \\
& \times\left[\left(\theta_{n n^{\prime}}^{2}+\mathrm{i} \theta_{n n^{\prime}}-1\right) \mathbf{I}-\left(\theta_{n n^{\prime}}^{2}+3 \mathrm{i} \theta_{n n^{\prime}}-3\right)(\hat{\mathbf{z}} \otimes \hat{\mathbf{z}})\right]
\end{aligned}
$$

where $\theta_{n n^{\prime}}=k\left|z_{n}-z_{n^{\prime}}\right|, \mathbf{I}$ is the $3 \times 3$ identity matrix, and $\otimes$ denotes the dyadic product.

Now, these dipoles give rise to a scattered electric far-field $\mathbf{E}^{\text {scat }}=\mathbf{f}(\hat{\mathbf{r}}) \mathrm{e}^{\mathrm{i} k r} / r$, where

$$
\mathbf{f}(\hat{\mathbf{r}})=k^{2} \sum_{n}\left[\mathbf{p}_{n}-\left(\mathbf{p}_{n} \cdot \hat{\mathbf{r}}\right) \hat{\mathbf{r}}\right] \mathrm{e}^{-\mathrm{i} k z_{n} \cos \theta}
$$

is the field amplitude for a direction of emission $\hat{\mathbf{r}}$ that forms an angle $\theta$ with the array. Calculating the far-field Poynting vector and dividing by the photon energy $\hbar \omega$, we finally obtain the emission probability ${ }^{22} \Gamma=\int_{0}^{\infty} d \omega \int d^{2} \hat{\mathbf{r}} \Gamma(\omega, \hat{\mathbf{r}})$, where

$$
\Gamma(\omega, \hat{\mathbf{r}})=\frac{1}{4 \pi^{2} \hbar k}|\mathbf{f}(\omega, \hat{\mathbf{r}})|^{2}
$$

is the doubly differential probability of emitting one photon per electron and per unit of frequency and solid-angle ranges.

\section{B. Lattice structures}

We consider three types of lattices: periodic, quasiperiodic, and hyperuniform, all of them with the same average distance between particles $d$. The set of lattices belong to each of these sets is a subset of the preceding one, but here, we focus on three specific realizations. Despite their very different short-range structure, they exhibit long-range correlations that translate into resonances in reciprocal space, and consequently, also into different angular SP emission patterns (see below).

The main property of hyperuniform lattices is the absence of long-wavelength fluctuations: this is mathematically equivalent to the vanishing of their structure factor for large 
distances (i.e., low wave vector, see Eq. (6) below) ${ }^{21 / 24}$. We study tessellated arrays as an example of one-dimensional hyperuniform arrangements. ${ }^{21}$ A tessellated array of $N$ particles is constructed by considering $N$ contiguous segments of length $d$ along the $z$ axis, and then placing one particle in each segment with a uniform random distribution along its length.

We also study Fibonacci arrays as a special case of one-dimensional quasicrystals. ${ }^{20}$ In these arrays there are two possible distances between contiguous particles, which we denote long $(L)$ and short $(S)$ ones. The $N$-particle array is constructed by starting with two particles separated by a distance $L$. Then, we iteratively apply the following pair of substitutions: every distance $L$ is substituted by a pair of distances $L S$ (i.e., with a new particle inserted), and every distance $S$ is transformed into $L$. This procedure is repeated until a number of particles $\geq N$ is obtained, and we then retain only the first $N$ particles. By construction, the ratio between $L$ and $S$ intervals contained in the array is the golden ratio $\phi=(1+\sqrt{5}) / 2,2$ so that the average nearest-neighbor distance is $d=(\phi L+S) /(\phi+1)$. In this study, we take $L=\phi S$.

\section{Simple analytical model based on momentum conservation}

Interestingly, the $z$ dependence of $\mathbf{E}^{\text {ext }}(z, \omega)$ [see Eq. (1)] comes only through a phase factor $\mathrm{e}^{\mathrm{i} \omega z / v}$. For a large periodic array of period $d$, this allows us to write

$$
\mathbf{p}_{n} \approx \mathrm{e}^{\mathrm{i} \omega z_{n} / v} \mathbf{p}
$$

where $\mathbf{p}$ is shared by all particles. Additionally, the far-field produced by these dipoles is proportional to $\sum_{n} \mathrm{e}^{\mathrm{i}\left(\omega / v-k_{\|}\right) d n}$, where $k_{\|}$is the projection of the light wave vector along $z$. In the limit of a large number of particles $(N \gg 1)$, this sum becomes $\approx(2 \pi / d) \sum_{m} \delta\left(k_{\|}-\right.$ $\omega / v-2 \pi m / d)$, leading to the well-known condition $m \lambda / d=\cos \theta-c / v$ for SP emission of order $m$ with wavelength $\lambda$ along an angle $\theta$ relative to the array direction ${ }^{1}$ Momentum conservation can thus involve transfers to the particle lattice associated with wave vectors $2 \pi m / d$.

The linear aperiodic arrays under consideration can also absorb momentum with certain preferential wave vectors that should correspond to lattice singularities in Fourier space. In order to determine those wave vectors, we analyze the structure-factor sum

$$
S_{N}\left(k_{\|}\right)=\sum_{n} \mathrm{e}^{\mathrm{i} k_{\|} z_{n}}
$$



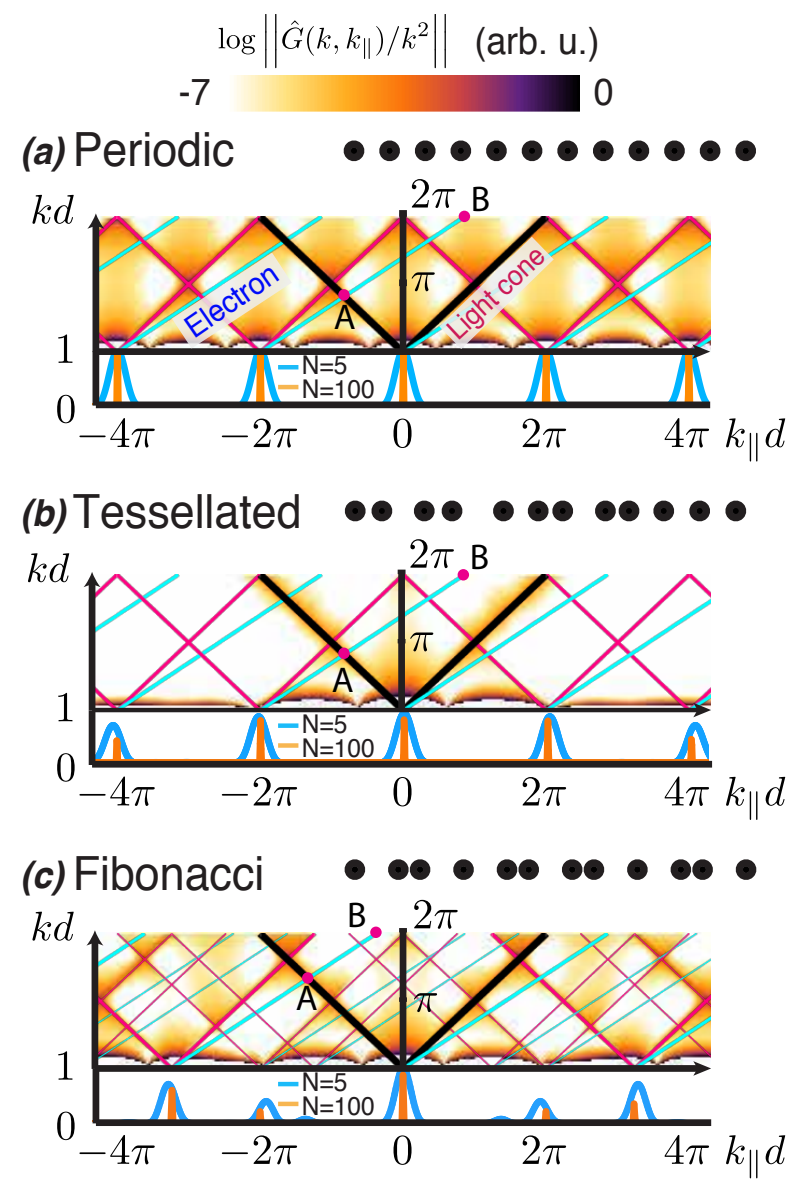

FIG. 2: Periodic and aperiodic arrays in real and reciprocal space. We consider three different types of arrays: (a) periodic, (b) tessellated, and (c) Fibonacci, all of them with $N=100$ particles. The average distance between sites is $d$ in all cases. For each type of array, we show examples of the particle distributions (black dots), as well as dispersion diagrams of the interparticle interaction [contour plots, $\left|\operatorname{det}\left\{G\left(k_{\|}, \omega\right)\right\} / \omega^{2}\right|$, Eq. (7)], and the lattice structure factors [lower plots, $\left|S_{N}\left(k_{\|}\right) / N\right|^{2}$, Eq. (6)]. The light cone (red lines), the electron line (blue lines), and their replicas, associated with displacements by wave vectors corresponding to the divergences of $S_{N}$, are shown in the dispersion diagrams.

Obviously, $S_{N}\left(k_{\|}\right)$diverges for periodic arrays when the parallel wave vector $k_{\|}$is a multiple of $2 \pi / d$, as shown in the lower part of $2\left(\right.$ a), which represents $\left|S_{N} / N\right|^{2}$ for two different values of $N$. Understandably, the tessellated lattice produces divergences at the same $k_{\|}$positions as in the periodic array (see Fig. 2(b), lower part), although their strength diminishes with increasing order, essentially as a result of hyperuniformity. ${ }^{21}$ In contrast, the Fibonacci 
lattice gives rise to sharp resonances associated with its two different characteristic lengths (see Fig. 2(c), lower part, where we observe peaks at multiples of $2 \pi / S$ and $2 \pi / L$ ).

A similar analysis can be performed to study the optical modes supported by the arrays. Inspired again by periodic structures, in which such modes are controlled by lattice resonances associated with the in-phase interaction between distant sites, as revealed by divergences in the Fourier transform of $\mathcal{G}, \frac{25}{2}$ we consider the sum

$$
G\left(k_{\|}, \omega\right)=\frac{1}{N} \sum_{n} \sum_{n^{\prime} \neq n} \mathcal{G}_{n n^{\prime}} \mathrm{e}^{\mathrm{i} k_{\|}\left(z_{n}-z_{n^{\prime}}\right)} .
$$

The color plots of Fig. 2 represent $\left|\operatorname{det}\left\{G\left(k_{\|}, \omega\right)\right\} / \omega^{2}\right|$ for the three types of lattices under consideration (with $N=100$ elements each). We observe that a central resonance occurs at the line cone $\left(k_{\|}=k\right)$, which is flanked by replicas that are displaced by wave vectors corresponding to the divergences of $S_{N}$.

We are now prepared to extend the kinematical condition of SP radiation to aperiodic arrays:

$$
k_{\|, j} \lambda / 2 \pi=\cos \theta-c / v
$$

where $j$ runs over the divergences of $S_{N}\left(k_{\|}\right)$. This condition is represented by blue lines in Fig. 2. We thus expect to find emission maxima at directions roughly determined by the crossings of those lines with the divergences of $G$. This picture must be however corrected by dynamical effects in the interaction between particles, which render maximum polarization at frequencies that are slightly shifted with respect to the noted condition. For a qualitative analysis of this effect, which turns out to be rigorous in periodic arrays, we can adopt Eq. (5) as an approximate relation also for aperiodic lattices. Upon insertion of this expression into Eq. (2), we find

$$
\mathbf{p} \approx \frac{1}{\alpha^{-1}-G\left(k_{\|}, \omega\right)} \cdot \mathbf{g}(\omega)
$$

Obviously, the condition for maximum polarization is $\operatorname{det}\left\{\alpha^{-1}-G\right\}=0$. Notice that $G$ receives individual dipole-dipole interaction contributions that scale as $1 /(\min \{d, \lambda\})^{3}$ with particle separation $d$ and emission wavelength $\lambda$, whereas $\alpha$ is typically of the order of the particle volume, unless strong particle resonances are excited. Therefore, $\alpha^{-1}$ can only be compensated by $G$ when the latter diverges as a result of coherent lattice interactions, as described in Fig. 2. The actual resonances of the array therefore depend on the type and 

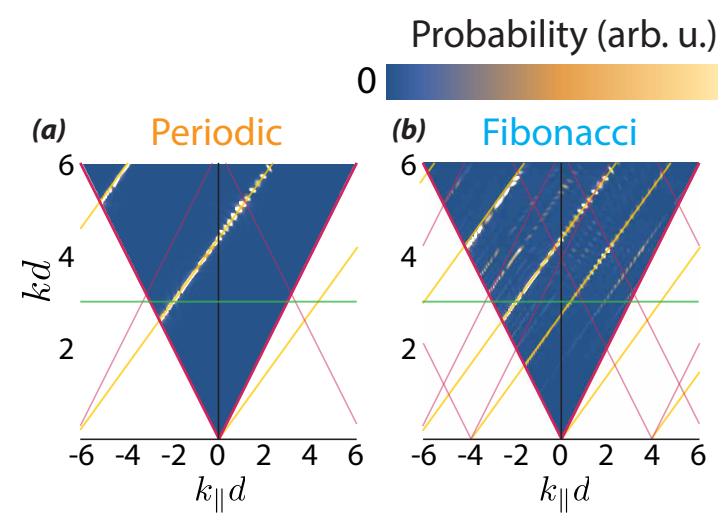

(e)

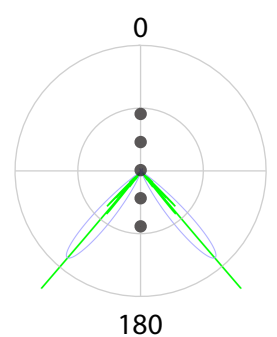

(f)

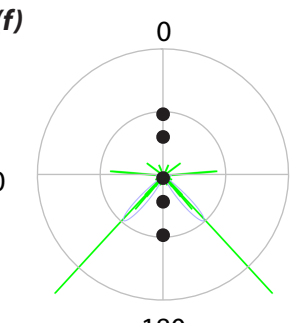

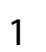

1

(c) Tessellated

(d) Intensity/

particle

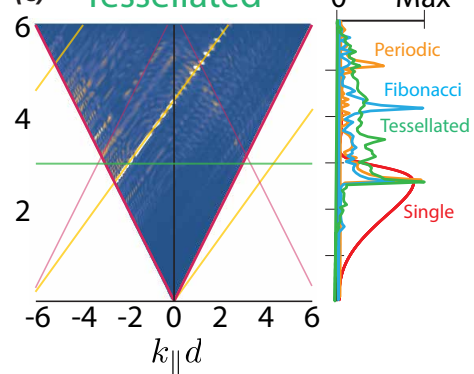

(g)

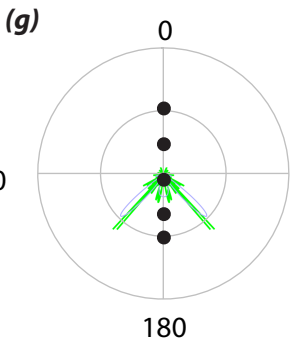

FIG. 3: Cathodoluminescence probability and angular emission profiles. (a-c) We show the SP emission probability as a function of photon wave vector $k$ and parallel wave vector $k_{\|}$ for the three different arrays considered in Fig. 2. The probability is calculated from Eq. (4) and represented in color scale for arrays consisting of $N=100$ particles. (d) Angle-integrated emission probability for the three types of arrays, as compared with the emission from a single particle. (e-g) Angular distribution of the emission for fixed frequency, as indicated by corresponding horizontal cuts shown in (a-c). We compare results for $N=20$ and $N=100$. The electron beam is passing at a distance $b=75 \mathrm{~nm}$ from the particle centers with energy $E \sim 200 \mathrm{keV}$ (i.e., $v \sim 0.7 c$ ). The average spacing is $d=120 \mathrm{~nm}$. The spheres have a diameter of $50 \mathrm{~nm}$ and a permittivity $\epsilon=12$.

size of the particles! $\frac{\sqrt{25}}{5}$ they are generally closer to the divergences of $G$ when the particles are smaller, and they are redshifted with respect to that condition when the real part of $\alpha$ is positive.

An approximate expression for the SP emission probability in the large $N$ limit is obtained by inserting Eq. (9) into Eq. (3), and this in turn into Eq. (4) to yield

$$
\Gamma(\omega, \hat{\mathbf{r}}) \approx \frac{k^{3}}{4 \pi \hbar}|\mathbf{p}-(\hat{\mathbf{r}} \cdot \mathbf{p}) \hat{\mathbf{r}}|^{2}\left|S_{N}\left(\omega / v-k_{\|}\right)\right|^{2}
$$

where $S_{N}$ is the structure factor defined in Eq. (6). 


\section{RESULTS AND DISCUSSION}

Figure 3 shows the emission probability for finite periodic and aperiodic arrays as a function of photon energy and angle of emission. The emission intensity is peaked around a frequency region in which each individual particle shows a prominent response [see Fig. 3(d)], which is associated with the first Mie mode for the $50 \mathrm{~nm}$ diameter silicon spheres $(\epsilon=12)$ under consideration. For reference, the average spacing between sphere centers is $d=120 \mathrm{~nm}$. As expected, the fine structure within this region is dominated by the crossings with the electron dispersion line and its replicas [Fig. 3(a-c), orange curves]. Additionally, the intensity is enhanced near the crossings of the line cone and its replicas. This is more clearly emphasized in the angular distribution patterns shown in Fig. 3(e-g). Remarkably, the angular patterns are still clearly identifiable when a relatively small number of particles $(N=$ 10) is considered in all three types of lattices. Incidentally, the periodic and the tessellated lattices both lead to the same angles of emission, although the former displays stronger features, in agreement with the results of Fig. 2. In contrast, the Fibonacci lattice produces a more involved angular pattern, which reveals a denser set of resonances in reciprocal space.

We put the analytical model described above to the test in Fig. 4, where we compare it with the full calculation of Eq. (4) for periodic and aperiodic arrays consisting of either $N=5$ or $N=100$ particles. Interestingly, both the periodic and the tessellated arrays are well reproduced by the analytical model, thus corroborating that the approximate phase relation of Eq. (5) constitutes a good ansatz. In contrast, the Fibonacci lattice is only qualitatively described by this approximation, which is an indication that the complex behavior of this type of lattice in reciprocal space cannot be well captured by such a simple phase relation.

A class of aperiodic structures is formed by randomly removing particles from an originally periodic array. This is explored in Fig. 5, which in this way also address the robustness of the SP emission features against imperfections of the array. Remarkably, the dominant emission feature is clearly resolvable even after removing $40 \%$ of the particles.

\section{CONCLUSION}

In summary, we have shown that sharp SP emission patterns are produced by en electron moving parallel to different types of one-dimensional particle arrays, including periodic and 


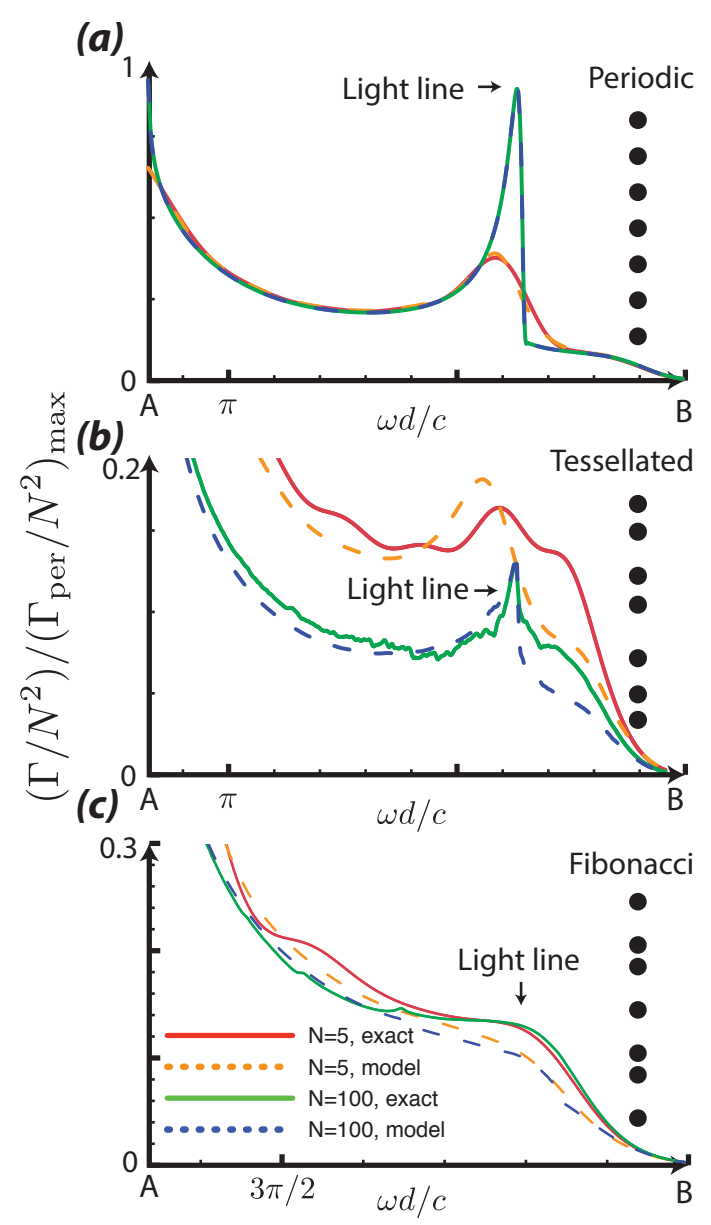

FIG. 4: Analytical description of SP emission in finite aperiodic arrays. We show the emission intensity for electrons moving parallel to finite arrays consisting of $N=5$ and $N=100$ particles with periodic (a) and aperiodic (b,c) arrangements. The emission energy and angle correspond to the $\mathrm{AB}$ segments indicated in Fig. 2

aperiodic arrangements. The condition for such intense patterns is that the lattice displays strong features in reciprocal space. We have shown examples of strong features in the Fourier transforms of periodic, quasiperiodic, and hyperuniform lattices, which lead to SP emission peaks associated with a generalized condition that relates the angle and frequency of the emission to the peak wave vectors in reciprocal space [see Eq. (8)]. A simple analytical model for the emission produces results in very good agreement with a more exact analysis, taking into account the interaction among particles and its dominant reciprocal-space features. 


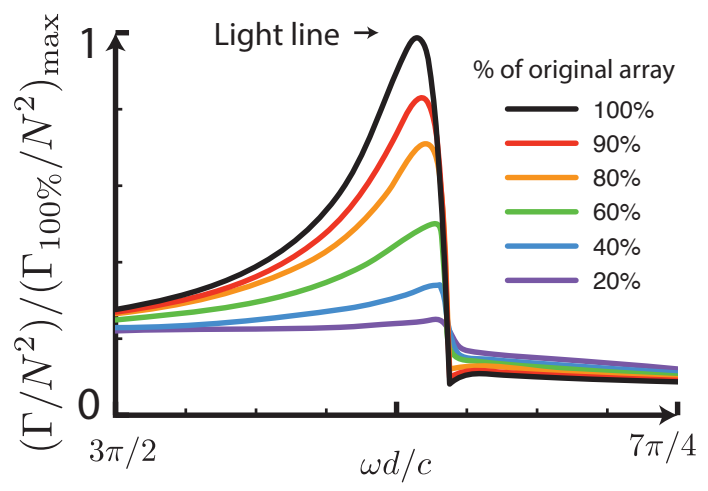

FIG. 5: Robustness of the SP emission features. We show the emission from an initially periodic array consisting of $N=1000$ particles in which a a fraction of them have been randomly removed. The emission is shown for different percentages of particles remaining. The spectral range covers the dominant right feature of Fig. 4(a).

\section{Acknowledgments}

This work has been supported in part by the the Spanish MINECO (MAT2014-59096-P and SEV-2015-0522) and AGAUR (Grants FI_B 00492-2015 and 2014 SGR 1400).

* Electronic address: javier.garciadeabajo@icfo.es

1 S. J. Smith and E. M. Purcell, Phys. Rev. 92, 1069 (1953).

2 O. Haeberlé, P. Rullhusen, J. M. Salomé, and N. Maene, Phys. Rev. E 49, 3340 (1994).

3 J. P. Bachheimer, Phys. Rev. B 6, 2985 (1972).

4 M. J. Moran, Phys. Rev. Lett. 69, 2523 (1992).

${ }^{5}$ G. Doucas, J. H. Mulvey, M. Omori, J. Walsh, and M. F. Kimmitt, Phys. Rev. Lett. 69, 1761 (1992).

${ }^{6}$ H. Ishizuka, Y. Kawamura, K. Yokoo, H. Shimawaki, and A. Hosono, Nucl. Instrum. Methods Phys. Res. A 445, 276 (2000).

7 R. Temkin, Science 280, 854 (1998).

8 S. E. Korbly, a. S. Kesar, J. R. Sirigiri, and R. J. Temkin, Phys. Rev. Lett. 94, 1 (2005).

9 W. Liu, Z. Yang, Z. Liang, D. Li, K. Imasaki, Z. Shi, F. Lan, and G. S. Park, Nucl. Instrum. 
Methods Phys. Res. A 580, 1552 (2007).

10 L. Schachter and A. Ron, Phys. Rev. A 40, 876 (1989).

11 D. Li, K. Imasaki, Z. Yang, G. S. Park, S. Miyamoto, S. Amano, and T. Mochizuki, Nucl. Instrum. Methods Phys. Res. A 572, 948 (2007).

12 J. Urata, M. Goldstein, M. F. Kimmitt, A. Naumov, C. Platt, and J. E. Walsh, Phys. Rev. Lett. 80, 516 (1998).

13 Y. Shibata, S. Hasebe, K. Ishi, S. Ono, M. Ikezawa, T. Nakazato, M. Oyamada, S. Urasawa, T. Takahashi, T. Matsuyama, et al., Phys. Rev. E 57, 1061 (1998).

14 D. Li, K. Imasaki, Z. Yang, and G. S. Park, Appl. Phys. Lett. 88, 201501 (2006).

15 N. Yamamoto, F. J. García de Abajo, and V. Myroshnychenko, Phys. Rev. B 91, 125144 (2015).

16 F. J. García de Abajo, Phys. Rev. E 61, 5743 (2000).

17 K. Ohtaka and S. Yamaguti, Optics and Spectroscopy 91, 506 (2001).

18 T. Ochiai and K. Ohtaka, Opt. Express 13, 7683 (2005).

19 S. Yamaguti, J. Inoue, O. Haeberlé, and K. Ohtaka, Phys. Rev. B 66, 195202 (2002).

20 E. Abe, Y. Yan, and S. J. Pennycook, Nat. Mater. 3, 759 (2004).

21 S. Torquato and F. H. Stillinger, Phys. Rev. E 68, 041113 (2003).

22 F. J. García de Abajo, Rev. Mod. Phys. 82, 209 (2010).

23 M. Abramowitz and I. A. Stegun, Handbook of Mathematical Functions (Dover, New York, 1972).

24 S. Torquato, G. Zhang, and F. Stillinger, PRX 5, 021020 (2015).

25 F. J. García de Abajo, Rev. Mod. Phys. 79, 1267 (2007). 